\title{
Integrando Emulador GNS3 como Suporte de Ensino na Disciplina de Redes de Computadores no Ambiente AVA
}

\author{
Isaias B. Silva ${ }^{1}$, Carla K. M. Marques ${ }^{2}$, Rommel W. Lima ${ }^{2}$ \\ ${ }^{1}($ UERN/UFERSA) - Programa de Pós-graduação em Ciência da Computação - (PPg-CC) \\ ${ }^{2}$ Departamento de Informática - Universidade do Estado do Rio Grande do Norte - (UERN) \\ Caixa Postal 70 - 59.610-190 - Mossoró - RN - Brazil. \\ \{isaias.ops, carla.katarina, rommel.lima\}@gmail.com
}

\begin{abstract}
With the advancement of Information Technology (IT) and the reality of data storage in the cloud local operating Systems and applications will tend to be embedded and virtualized to meet cost and practical needs by instituitions and users respectively. This work proposes to integrate the local GNS3 Emulator in a Virtual Learning Environment with the objective of supporting the practical activities during the discipline of computer networks in Distance Learning (DL) courses.
\end{abstract}

Resumo. Com o avanço da Tecnologia da Informação (TI) e da realidade do armazenamento de dados na nuvem, os sistemas operacionais e aplicações locais tenderão a ser incorporados e virtualizados para atender necessidades de custo e praticidade por instituições e usuários respectivamente. Este trabalho propõe integrar o Emulador GNS3 local em ambiente Virtual de Aprendizagem com objetivos de dar suporte nas atividades práticas durante a disciplina de redes de computadores nos cursos EaD (Ensino a Distancia).

\section{Introdução}

Os recursos tecnológicos tem evoluído rapidamente nas últimas décadas. Os avanços por meio da Internet tem trazido oportunidades para o desenvolvimento de atividades a distancia, favorecendo a expansão da educação a distancia. Nesse caso, as TIC's tem contribuído de maneira significativa para o redimensionamento das estratégias de ensinar e aprender, proporcionando diferentes ferramentas ou metodologias para o apoio de aprendizagem (Amaral 2011).

Os AVAS são propícios para o desenvolvimento de atividades, oferecendo um espaço repleto de interatividade e recursos que podem ser usados no meio acadêmico, enquanto que os simuladores são ferramentas de simulação que promovem o acesso a experimentos a partir de um espaço virtual, compensando a falta de interação e a indisponibilidade de horários ou de recursos necessários às experiências práticas (Arieira 2009).

Alguns trabalhos existentes na literatura como o do Ferreira (2014) e Petcu et al (2013) abordam essa temática de adaptação e ou ambientação de simuladores de redes de computadores para a prática de cenários previamente simuláveis em AVA. Diferentemente de outras ferramentas existentes como o NS2 e o Packet Tracer, o 
simulador de redes GNS3 (2017) possui como característica principal adicionar imagens de sistemas operacionais dos dispositivos reais a um ambiente de emulação virtual, além de contribuir para que os testes se tornem mais próximos das experiências realizadas em ambiente real. Apesar dessa vantagem, essa ferramenta funciona atualmente apenas em ambiente local ou desktop.

Visando contribuir para melhorar o ensino/aprendizagem em disciplinas de redes de computadores por meio da implementação de ferramentas em AVA, este trabalho apresenta uma adaptação da ferramenta de Emulação GNS3 a ser implantada no AVA através da versão GNSMOOD e possui como principais características a portabilidade, podendo ser acessada via web, a facilidade de configuração das componentes, bem como, o armazenamento de projetos na nuvem.

\section{Laboratório virtual de redes GNSMOOD}

O GNSMOOD é um laboratório virtual de redes de computadores portado ao Ambiente Virtual de Aprendizagem (AVA) do MOODLE sendo portanto, uma adaptação da ferramenta GNS3 para $W E B$ e atualmente trabalha num ambiente local ou desktop. A ideia de desenvolver o GNSMOOD surgiu devido a dificuldades quanto a limitação local ou desktop e de hardware para se processar a ferramenta durante as aulas de redes, bem como a complexidade de se gerenciar recursos dos objetos simuláveis durante processo de emulação.

Esse laboratório permite tornar a criação de cenários de redes de computadores o mais prático possível além da possibilidade de adicionar e gerenciar a imagem IOS (Internetwork Operating System) de roteadores e máquinas virtuais, sendo esta a principal característica que a diferencia de outras ferramentas do gênero voltadas especificamente ao AVA.

\subsection{Interface $G U I$ :}

A interface $G U I$ da ferramenta virtual comparada a interface da ferramenta GNS3 para Desktop é mais interativa. A Figura 1, apresenta uma visão da aplicação GNSMOOD implementada em HTML5 e Javascript.

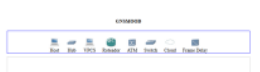

\section{Figura 1. Interface GNSMOOD}

Principais funcionalidades da ferramenta:

- VPCS - ou Virtual PC Simulator, permite criar PCs virtuais ex: QEMU;

- Hub, Switch e ATM - usado para interligar dispositivos na rede;

- Roteador - O usuário poderá adicionar uma imagem IOS de roteadores Cisco para executar a partir deste, como se fosse real;

- Cloud - cria uma conexão local de redes usando: IP, DNS, GATEWAY e NAT; 
VI Congresso Brasileiro de Informática na Educação (CBIE 2017)

Anais do XXVIII Simpósio Brasileiro de Informática na Educação (SBIE 2017)

- Salvar-Salva projeto GNSMOOD na nuvem.

\subsection{Arquitetura:}

Após a configuração entre o servidor e a aplicação GUI do GNSMOOD o processo de integração desse laboratório ao ambiente virtual de aprendizagem faz uso da API Learning Tools Interoperability (LTI) a qual possibilita integrar aplicações hospedadas remotamente. A Figura 2 exemplifica a implantação do laboratório virtual de redes de computadores ao ambiente virtual de aprendizagem Moodle.

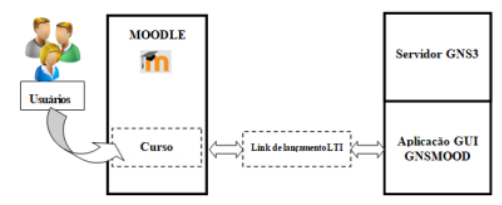

Figura 2. Arquitetura GNSMOOD

A disponibilização desse laboratório no AVA é feita por intermédio dos tutores da disciplina de redes de computadores ao compartilhar entre os alunos que estão inseridos nesse mesmo ambiente virtual por meio do link fornecido pelo LTI.

\section{Materiais e Métodos}

A interface do laboratório de simulação GNSMOOD está sendo implementada em Javascript usando recursos do HTML5 que possui algumas bibliotecas ao auxiliar no processo de aprimoramento da ferramenta virtual. Dentro do HTML5 está presente o pacote do $C A N V A S$ essencial para a implantação do drag and drop de objetos na área simulável do GNSMOOD, bem como, dentre outros recursos gráficos como visto na Figura 1. Enquanto no back-end o servidor trabalha fornecendo a comunicação de parâmetros de objetos presentes na aplicação. O processo de requisição e envio de dados durante a sincronização entre a criação do projeto do usuário e o servidor faz uso da biblioteca CuRL juntamente com a linguagem de notação JSON. Para garantir a estabilidade da ferramenta, será disponibilizado servidores externos ao AVA para atender a demanda de processamento da aplicação por parte dos usuários.

\section{Referências}

Amaral, E. M. H., Avila, B., Zednik, H. e Tarouco, L. (2011) "Laboratório Virtual de Aprendizagem: Uma proposta Taxonômica", In: Revista Novas Tecnologias na Educação RENOTE, Brasil.

Arieira, J. O., Arieira, C. R. D.; Fusco, J. P. A.; Sarcomano, J. B.; e Bertega, M. O. P. (2009) “Avaliação do Aprendizado Via Educação a Distancia: A Visão dos Discentes.”, In: Scielo, Brasil.

Ferreira, K. H. A. (2014) "Integrando o Simulador 2.0 a um Ambiente Virtual de Aprendizagem”, Tese de Mestrado, UERN/UFERSA, Brasil.

Petcu, D.; Iancu, B.; Peculea, A.; Dadarlat, V. e Cebuc, E. (2013), "Integrating Cisco Packet Tracer With Moodle Platform: Support for Teaching and Automatic Evaluation", In: IEEE.

GNS3, (2017). “GNS3 2.0 Documentation”, http://api.gns3.net/en/2.0/general.html\#architecture, fevereiro. 